\title{
RELACIONES ENTRE ÉTICA PROFESIONAL Y DESEMPEÑO LABORAL EN PROFESORES DE UN DISTRITO DEL CONO NORTE DE LIMA
}

\author{
Relations between professional ethic and labour performance in \\ teachers of a district of the Cono Norte of Lima \\ Manuel Torres Valladares ${ }^{1}$, Rosario Lajo Lazo \\ Universidad Nacional Mayor de San Marcos, Lima, Perú \\ (RECIBIDO EL 05/04/2011 - AcEPTADo EL 09/06/2011)
}

\begin{abstract}
RESUMEN
El propósito fundamental del presente trabajo de investigación fue obtener una descripción de cómo percibe el docente su ética profesional y cómo este se relaciona con su desempeño laboral. Este tema es trascendente en tanto estamos en una época en la cual se dice que se han perdido los valores. Se busca, por tanto, propiciar que estos sean retomados en el aula para que los estudiantes, al egresar, sean personas comprometidas no solo con su quehacer profesional sino también con su entorno: su comunidad, su país y el planeta mismo, y en este aspecto la ética y el quehacer profesional del docente son fundamentales.

En el desarrollo de la presente investigación se utilizó el Inventario de comportamiento ético docente de Ana Hirsch Adler y el inventario de desempeño laboral de Manuel Torres. Los análisis estadísticos a los que fueron sometidas las pruebas nos indican que los instrumentos son validos y confiables. Estas pruebas fueron aplicadas a 304 docentes de un distrito de Lima. Los resultados estadísticos nos indican que la ética profesional se encuentra relacionada significativamente al desempeño laboral de los docentes, así mismo se encontró que existen diferencias entre los docentes de las instituciones educativas de la muestra respecto de las variables en estudio.
\end{abstract}

Palabras clave: Ética, desempeño laboral, calidad educativa, valores.

\begin{abstract}
The fundamental purpose of the current investigation work was to obtain a description of how the teacher percibes his professional ethic and how this relates with his labour performance. This issue is trascendental as we are in a time in which it is said the values have been lost. For instance, we are looking foward to propiciate that these can be retaken at the classroom so the students, when they graduate, are comitted people not only with their professional tasks but also with their environment: their community, country and the planet itself and in this aspect, the ethic and the professional tasks of the teacher are fundamental.
\end{abstract}

1 Docente Principal de la Facultad de Psicología UNMSM. E-mail: mtorresv@unmsm.edu.pe 
In the development of the current investigation, the Inventory of Teacher's Ethical Behaviour of Ana Hirsch Adler and the Inventory of Labour Performance of Manuel Torres were used. The statistical analyses the tests were submitted to indicate us that the instruments are valid and reliable. These tests were taken to 304 teachers of a district of Lima. The statistical results indicate us that the professional ethic is significantly related to labour performance of the teachers. Moreover, it was found that signifcant differences exist between the teachers of the educative instutions of the sample regarding the variables in study.

Keywords: Ethic, labour performance, educative quality, values.

\section{INTRODUCCIÓN}

En el mundo de hoy, cuando los procesos de globalización de los mercados, el acelerado desarrollo de la ciencia y tecnología, nos plantean grandes desafíos, la institución educativa tiene la necesidad de adaptar sus sistemas y modelos funcionales para no quedar de espalda a los nuevos requerimientos de la sociedad. En este proceso de cambio el trabajo que desempeñan los docentes viene a ser fundamental, porque son ellos los profesionales que tienen como tarea no solo contribuir en el desarrollo intelectual y social de sus alumnos, sino también en su formación ética. Actualmente, la ética es un elemento que se considera necesario impulsar en la formación de todo profesional para garantizar su ejercicio responsable y comprometido con el bienestar de la sociedad, por lo que debe ser obligatoria su incorporación en el currículo de todas las facultades de la Universidad.

En el mundo de hoy las instituciones educativas tienen significativas funciones sociales y culturales en la construcción de la sociedad, sobre todo cuando buscan modos diversos de disminuir la inequitativa distribución de la riqueza, promover la movilidad social y estudiar y formular opciones de solución para problemas prioritarios. Los valores y el comportamiento ético son parte de estos asuntos.

Las profesiones y los profesionales, de todas las áreas del conocimiento, ocupan un lugar significativo en el mundo social, pues aportan bienes y servicios que requieren la propia sociedad. Su desempeño y actuación están siempre en la mira de los sectores, grupos e individuos (a nivel local, regional, nacional e internacional). El comportamiento ético es parte intrínseca de la profesión y del sentido y proyectos de vida de los sujetos. Constituye, además, junto con la competencia profesional y técnica, lo que las personas mejor pueden apreciar de su labor.

Hoy se vive una especial sensibilidad y demanda social de ética con respecto a los profesionales. Se insiste con mayor frecuencia en la importancia de incorporar elementos éticos en su formación y en el ámbito de investigación científica y socio-cultural. En esta línea se toma como muy importante la formación ética de los docentes en tanto son ellos los que tienen la responsabilidad, a nivel institucional, de formar a los futuros ciudadanos de la nación.

En la escuela se establecen relaciones morales entre profesores y alumnos que se manifiestan de muchas formas, por ejemplo, cuando se organiza el trabajo escolar se asumen posturas que pueden ir desde la indiferencia hasta la hostilidad, tanto por parte de los maestros hacia los estudiantes como de estos hacia sus pares. Sin duda el profesor manifiesta en el 
salón de clases su ser moral, su interioridad queda descubierta en cada acción reiterada y continua que asume frente a sus alumnos que le dan su sello personal, su identidad y su consistencia profesional, razones por las que es conocido por sus estudiantes.

Cada día aumenta la preocupación por saber qué sucede en las aulas, ya que es el espacio donde los niños y jóvenes pasan gran parte de su tiempo; porque además en la escuela los valores éticos alcanzan una expresión concreta que más tarde se expresarán en otros espacios; en ella se imprime ese 'modo de ser' del alumno, la persona se construye a sí misma, la moral se hace efectiva. Por otra parte, existe también un interés manifiesto por encontrar respuesta a los problemas morales presentes en la sociedad actual desde la escuela. "La escuela debe ser un espacio de participación democrática sensible a lo que pasa en el mundo, donde se afrontan los conflictos analizándolos críticamente, tratando de comprender sus causas reales desde una perspectiva global e intercultural adoptando compromisos para actuar en su resolución" (Gonzales 2000).

Apelar a la ética en la escuela, es apelar a la razón del profesor, descubrir que está obligado a introducir una actitud tolerante a la medida de sus alumnos. La posibilidad de reconocerlo y aceptarlo obliga al docente a revisar su conciencia, su ley moral, como fuente de su comportamiento en el aula. Es la razón, frente a las conductas caóticas de la violencia, el enojo y la indiferencia, que a veces adopta el profesor, o bien cuando toma más en cuenta los intereses de un estudiante sin tomar en cuenta los de otro, la que permite escapar del autoritarismo subjetivo, de valorar lo que sí vale dentro del salón de clases, de aquello que no se puede aceptar por el solo hecho de responder a su criterio de adulto; ayuda a reconocer al otro como fuente de las decisiones escolares.

Recurrir a la ética en la escuela es importante porque se requiere que el profesor construya un espacio dotado de 'sentido' en función de los valores culturales y espirituales que ayuden a construir la responsabilidad moral que la sociedad demanda del joven que se educa; salir de la inmediatez, de lo superfluo y el egoísmo para entrar a un mundo intersubjetivo, de compromiso con los otros. "El hombre es un ser social, la persona sólo puede constituirse en tanto que tal, en la relación interpersonal y en la relación social, y la estructura personal está tejida, por decirlo así, de interpersonalidad o intersubjetividad y de socialidad, del mismo modo que la conciencia moral es fuero interno, en tanto que fuero externo social, sí, pero interiorizado" (Aranguren, 1991).

Es importante señalar que la ética encierra en sí misma la noción de deber, en nuestro caso el respeto a los códigos morales que tienen como propósito orientar las actitudes docentes en favor del estudiante. Desde esta perspectiva tiene el deber de asegurar el derecho que tienen los estudiantes: el desarrollo pleno de sus potencialidades a partir de sus personas. En la medida en que el profesor cumple con ese deber asegura el derecho de sus alumnos y es que a todo deber del docente corresponde algún derecho del alumno y a todo derecho del alumno corresponde algún deber del docente.

Morin (2000) opina que en el nuevo milenio la ética debe formarse en las mentes, partir de la conciencia de que el ser humano es una trilogía, es al mismo tiempo: individuo, parte de una sociedad y de una especie humana, relacionando así la ética a la nueva dimensión de la era planetaria y sobrepasando la frontera de la conciencia individual. 
Es por eso que el hombre de esta época, afligido por los conflictos entre valores contrapuestos, piensa que la única validez es la decisión de la mayoría sin embargo es importante considerar que aún cuando se vive bajo el contexto de amenazas globales que hacen peligrar como nunca a la humanidad, sí existe la posibilidad de orientar este momento histórico desde una perspectiva ética a través de la interconexión con la educación y la globalización, donde la ética respalda y ratifica a la educación para coprotagonizar el más profundo esclarecimiento de muchos problemas humanos. Es por ello que la educación ejerce su poder transformador, a través de la actuación y vocación de servicio del docente, quien ejerce la función de mayor relevancia en el proceso educativo, por lo que la calidad del trabajo docente es una exigencia en el tiempo actual.

Como consecuencia de lo expuesto, los problemas de la presente investigación se plantean de la siguiente manera:

- ¿Qué relación existe entre la ética profesional y el desempeño laboral de los docentes de las Instituciones Educativas de un distrito del cono norte de Lima?

- ¿Qué diferencias existen en la ética profesional entre los docentes varones y mujeres?

- ¿Qué diferencias existen en el desempeño laboral entre los docentes varones y mujeres?

- ¿Qué diferencias existen entre los docentes, considerando su rango de edad, respecto de su ética profesional?

- ¿ Qué diferencias existen entre los docentes, considerando su rango de edad, respecto de su desempeño laboral docente?

- ¿Cuáles son los niveles de ética profesional que presentan los docentes de las Instituciones Educativas de un distrito del cono norte de Lima?

- ¿Cuáles son los niveles de desempeño laboral que presentan los docentes de las Instituciones Educativas de un distrito del cono norte de Lima?

\section{OBJETIVOS}

1. Establecer la relación que existe entre la ética profesional y el desempeño laboral de los docentes de las Instituciones Educativas de un distrito del cono norte de Lima.

2. Determinar las diferencias que existen en la ética profesional entre los docentes varones y mujeres.

3. Determinar las diferencias que existen en el desempeño laboral entre los docentes varones y mujeres.

4. Establecer las diferencias que existen entre los docentes, considerando su rango de edad, respecto de su ética profesional.

5. Establecer las diferencias que existen entre los docentes, considerando su rango de edad, respecto de su desempeño laboral docente.

6. Establecer los niveles de ética profesional que presentan los docentes de las Instituciones Educativas de un distrito del cono norte de Lima. 
7. Describir los niveles de desempeño laboral que presentan los docentes de las Instituciones Educativas de un distrito del cono norte de Lima.

\section{HIPÓTESIS}

1. Existe una relación significativa entre la ética profesional y el desempeño laboral de los docentes de las Instituciones Educativas de un distrito del cono norte de Lima.

2. Existen diferencias significativas entre los docentes varones y mujeres respecto de su ética profesional.

3. Existen diferencias significativas entre los docentes varones y mujeres respecto de su desempeño laboral.

4. Existen diferencias significativas entre los docentes, considerando su rango de edad, respecto de su ética profesional.

5. Existen diferencias significativas entre los docentes, considerando su rango de edad, respecto de su desempeño laboral docente.

\section{MÉTODOS Y RESULTADOS}

\section{Método}

El método de investigación utilizado fue el método descriptivo; ya que este describe las situaciones y eventos, además mide diversos aspectos del fenómeno a investigar. El estudio descriptivo se desarrolla describiendo las situaciones y eventos, es decir cómo se manifiesta el fenómeno a investigar, ya que este busca especificar las propiedades importantes del problema en cuestión. El método descriptivo mide independientemente los conceptos y también pueden ofrecer la posibilidad de predicciones aunque sean muy rudimentarias (Sánchez y Reyes, 2003).

\section{Diseño de investigación}

Para la selección del diseño de investigación se ha utilizado como base el libro de Hernández, Fernández y Baptista (2010) titulado Metodología de la Investigación. Según estos autores, el diseño adecuado para esta investigación es de tipo descriptivo correlacional.

$\mathrm{Su}$ figura representativa es la siguiente:

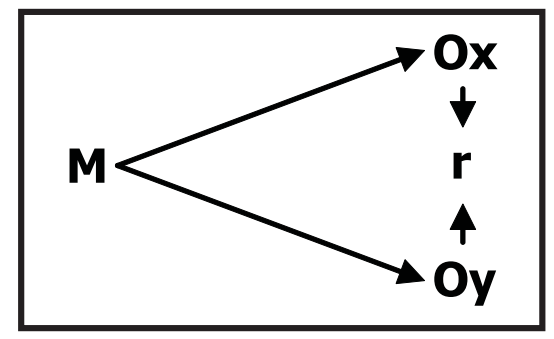


$\mathbf{M}=$ Muestra de investigación

$\mathbf{O x}, \mathbf{O y}=$ Observaciones de las variables

$\mathbf{r}=$ Relaciones entre variables

En este caso, se quiere correlacionar las variables la ética profesional y el desempeño laboral de los docentes de las Instituciones Educativas de un distrito del cono norte de Lima.

\section{MUESTRA}

La muestra de estudio que utilizamos, en tanto se ajusta a nuestras necesidades, es una muestra no probabilística de tipo intencionado, en la medida que es el investigador quien ha determinado de manera intencional el lugar y la muestra con la que se trabajó. Esta decisión se tomó considerando que aplicar los instrumentos es una tarea muy complicada, puesto que los docentes colaboran muy poco, sin embargo creemos que la muestra tomada es bastante representativa.

Se tomó como muestra docentes de nueve instituciones educativas que en total suman 304, con edades que fluctúan entre 25 y 55 a más años de edad. También se aprecia que la muestra está conformada mayoritariamente por mujeres $68.4 \%$, frente al $31.6 \%$ de los varones.

TABLA 1. Composición general de la muestra

\begin{tabular}{lcc}
\hline Sexo & $\mathrm{F}$ & $\%$ \\
\hline Masculino & 96 & 31.6 \\
Femenino & 208 & 68.4 \\
\hline Nivel & & \\
\hline Inicial & 9 & 3.0 \\
Primaria & 162 & 53.3 \\
Secundaria & 133 & 43.8 \\
\hline
\end{tabular}

\section{Instrumentos}

Los instrumentos utilizados en el desarrollo de la presente investigación fueron, en primer lugar, la Escala de Ética Profesional elaborado por Ana Hirsch Adler y compuesto por cuatro subescalas: Respeto al alumnado y responsabilidad docente, Honestidad y compromiso ciudadano, Formación ética y Confianza en el alumnado.

Igualmente se utilizó el Inventario de Desempeño Laboral, de Manuel Torres, compuesto por cinco subescalas: Saber conocer, Saber Hacer, Saber ser, Saber convivir y Saber emprender.

En ambos casos los resultados son calificados sobre la base de una escala Likert de cinco puntos, los cuales permitían expresar en términos de frecuencia la ocurrencia de los aspectos indicados y fluctúan desde Muy en desacuerdo (1) para el menor valor, hasta Muy de Acuerdo (5) para la mayor valoración. 


\section{Análisis de validez y confiabilidad de los instrumentos}

En la medida de que se necesita garantizar la idoneidad de los instrumentos de evaluación, se procedió a realizar los análisis estadísticos respectivos que nos indiquen sus niveles de validez y confiabilidad. El análisis psicométrico de la Escala de Ética Profesional indica que sus subescalas alcanzan alfa de cronbach que fluctúan entre 0.91 y 0.94 , obteniendo además un alfa de Cronbach para toda la prueba de 0.95 .

El análisis psicométrico del inventario de desempeño laboral docente indica que sus escalas alcanzan alfa de Cronbach que fluctúan entre 0.84 y 0.91 y la prueba total alcanza el 0.96 , lo cual indica que el instrumento es confiable.

Los análisis de la Validez de los instrumentos, realizado a través del Análisis factorial Exploratorio nos muestran que las pruebas presentan validez de Constructo.

\section{RESULTADOS}

TABLA 2. Análisis de la Bondad de Ajuste de la Curva Normal de las Variables estudiadas

\begin{tabular}{lccc}
\hline \multicolumn{1}{c}{ Variable } & Media & D.S. & K-S Z \\
\hline Respeto al alumnado & 18.62 & 8.69 & 4.12 \\
Honestidad & 14.68 & 6.25 & 4.39 \\
Formación ética & 13.35 & 6.25 & 3.37 \\
Confianza en el alumnado & 8.86 & 4.75 & 3.98 \\
& & & \\
Saber Conocer & 16.92 & 4.21 & 1.76 \\
Saber Hacer & 15.83 & 4.52 & 1.71 \\
Saber Convivir & 15.59 & 4.42 & 1.87 \\
Saber Ser & 16.46 & 4.93 & 1.86 \\
Saber Emprender & 17.83 & 4.76 & 1.76 \\
\hline
\end{tabular}

$\mathrm{n}=304$

Los resultados del análisis exploratorio de los datos (ver Tabla 2), en lo que se refiere a la forma de distribución, efectuado a través del test de bondad de ajuste a la curva normal de Kolmogorov-Smirnov, indica que en todas las variables estudiadas, se obtienen coeficientes K-S Z que son estadísticamente significativos, por lo que se puede concluir que no se presentan adecuadas aproximaciones a la curva normal, por lo que es factible utilizar para el análisis de los datos, estadísticas no paramétricas. 
TABLA 3. Análisis de Correlación (Rho de Spearman) entre Ética Profesional y Desempeño Laboral

\begin{tabular}{lcccccc}
\hline Variables & Conocer & Hacer & Convivir & Ser & Emprend & TCD \\
\hline Respeto al alumnado & $0.35^{* * *}$ & $0.43^{* * *}$ & $0.49^{* * *}$ & $0.40^{* * *}$ & $0.46^{* * *}$ & $0.48^{* * *}$ \\
Honestidad & $0.47^{* * *}$ & $0.61^{* * *}$ & $0.55^{* * *}$ & $0.54 * * *$ & $0.58^{* * *}$ & $0.62 * * *$ \\
Formación ética & $0.29^{* *}$ & $0.28^{* *}$ & $0.31^{* *}$ & $0.31^{* *}$ & $0.39 * * *$ & $0.36^{* * *}$ \\
$\begin{array}{l}\text { Confianza en el } \\
\text { alumnado }\end{array}$ & $0.34 * *$ & $0.41^{* * *}$ & $0.48^{* * *}$ & $0.42^{* * *}$ & $0.45^{* * *}$ & $0.48^{* * *}$ \\
\hline Total EPD & $0.42^{* * *}$ & $0.50^{* * *}$ & $0.53^{* * *}$ & $0.47^{* * *}$ & $0.55^{* * * *}$ & $0.56^{* * *}$ \\
\hline
\end{tabular}

$* \mathrm{p}<.05 * * \mathrm{p}<.01 * * * \mathrm{p}<.001$

$\mathrm{n}=304$

El análisis de las correlaciones entre la ética profesional y el desempeño docente indica que existen correlaciones significativas y positivas en todos los casos incluidos los totales de las variables en estudio.

TABLA 4. Prueba U de comparación de los puntajes de la Prueba de Ética Profesional por Sexo

\begin{tabular}{|c|c|c|c|c|c|c|}
\hline Variable & Sexo & $\mathrm{N}$ & $\begin{array}{l}\text { Rango } \\
\text { promedio }\end{array}$ & $\begin{array}{c}\text { U de } \\
\text { Mann-Whitney }\end{array}$ & $\mathrm{Z}$ & $\begin{array}{l}\text { Sig. } \\
\text { asintót. }\end{array}$ \\
\hline \multirow{2}{*}{ Respeto al alumnado } & Masculino & 96 & 145.47 & \multirow{2}{*}{9309.500} & \multirow{2}{*}{-.949} & \multirow{2}{*}{.343} \\
\hline & Femenino & 208 & 155.74 & & & \\
\hline \multirow{2}{*}{ Honestidad } & Masculino & 96 & 142.33 & \multirow{2}{*}{9007.500} & \multirow{2}{*}{-1.377} & \multirow{2}{*}{.169} \\
\hline & Femenino & 208 & 157.19 & & & \\
\hline \multirow{2}{*}{ Formación ética } & Masculino & 96 & 140.60 & \multirow{2}{*}{8842.000} & \multirow{2}{*}{-1.609} & \multirow{2}{*}{.108} \\
\hline & Femenino & 208 & 157.99 & & & \\
\hline \multirow{2}{*}{$\begin{array}{l}\text { Confianza en el } \\
\text { alumnado }\end{array}$} & Masculino & 96 & 147.03 & \multirow{2}{*}{9458.500} & \multirow{2}{*}{-.745} & \multirow{2}{*}{.456} \\
\hline & Femenino & 208 & 155.03 & & & \\
\hline \multirow{2}{*}{ Total EPD } & Masculino & 96 & 142.11 & \multirow{2}{*}{8986.500} & \multirow{2}{*}{-1.401} & \multirow{2}{*}{.161} \\
\hline & Femenino & 208 & 157.30 & & & \\
\hline
\end{tabular}

$* \mathrm{p}<.05 * * \mathrm{p}<.01 * * * \mathrm{p}<.001$

$\mathrm{n}=304$

El análisis de la diferencias entre los docentes varones y mujeres, realizado a través de la U de Mann-Whitney, indica que no existen diferencias estadísticas significativas en caso alguno por lo que podemos pensar que tanto los docentes varones como las mujeres tienen la misma opinión respecto de su desempeño laboral. 
TABLA 5. Prueba $\mathrm{Z}$ de comparación de medias de los puntajes de la Prueba de Desempeño Laboral por Sexo

\begin{tabular}{|c|c|c|c|c|c|c|}
\hline Variable & Sexo & $\mathrm{N}$ & $\begin{array}{l}\text { Rango } \\
\text { promedio }\end{array}$ & $\begin{array}{c}\text { U de Mann- } \\
\text { Whitney }\end{array}$ & $\mathrm{Z}$ & $\begin{array}{l}\text { Sig. } \\
\text { asintót. }\end{array}$ \\
\hline \multirow{2}{*}{ Saber Conocer } & Masculino & 96 & 143.56 & \multirow{2}{*}{9126.000} & \multirow{2}{*}{-1.209} & \multirow{2}{*}{.227} \\
\hline & Femenino & 208 & 156.63 & & & \\
\hline \multirow{2}{*}{ Saber Hacer } & Masculino & 96 & 138.99 & \multirow{2}{*}{8687.500} & \multirow{2}{*}{-1.826} & \multirow{2}{*}{.068} \\
\hline & Femenino & 208 & 158.73 & & & \\
\hline \multirow{2}{*}{ Saber Convivir } & Masculino & 96 & 155.29 & \multirow{2}{*}{9716.000} & \multirow{2}{*}{-.378} & \multirow{2}{*}{.706} \\
\hline & Femenino & 208 & 151.21 & & & \\
\hline \multirow{2}{*}{ Saber Ser } & Masculino & 96 & 144.98 & \multirow{2}{*}{9262.500} & \multirow{2}{*}{-1.016} & \multirow{2}{*}{.310} \\
\hline & Femenino & 208 & 155.97 & & & \\
\hline \multirow{2}{*}{ Saber Emprender } & Masculino & 96 & 142.74 & \multirow{2}{*}{9047.500} & \multirow{2}{*}{-1.318} & \multirow{2}{*}{.187} \\
\hline & Femenino & 208 & 157.00 & & & \\
\hline \multirow{2}{*}{ Total Desempeño } & Masculino & 96 & 144.08 & \multirow{2}{*}{9176.000} & \multirow{2}{*}{-1.135} & \multirow{2}{*}{.257} \\
\hline & Femenino & 208 & 156.38 & & & \\
\hline
\end{tabular}

$* \mathrm{p}<.05 * * \mathrm{p}<.01 * * * \mathrm{p}<.001$

$\mathrm{n}=304$

El análisis de la diferencias entre los docentes varones y mujeres, realizado a través de la U de Mann-Whitney indica que no existen diferencias estadísticas significativas en caso alguno por lo que podemos pensar que tanto los docentes varones como las mujeres tienen la misma opinión respecto de su desempeño laboral.

TABLA 6. Análisis de Varianza por Rangos de Kruskal - Wallis de la Ética Profesional por Rango de Edad

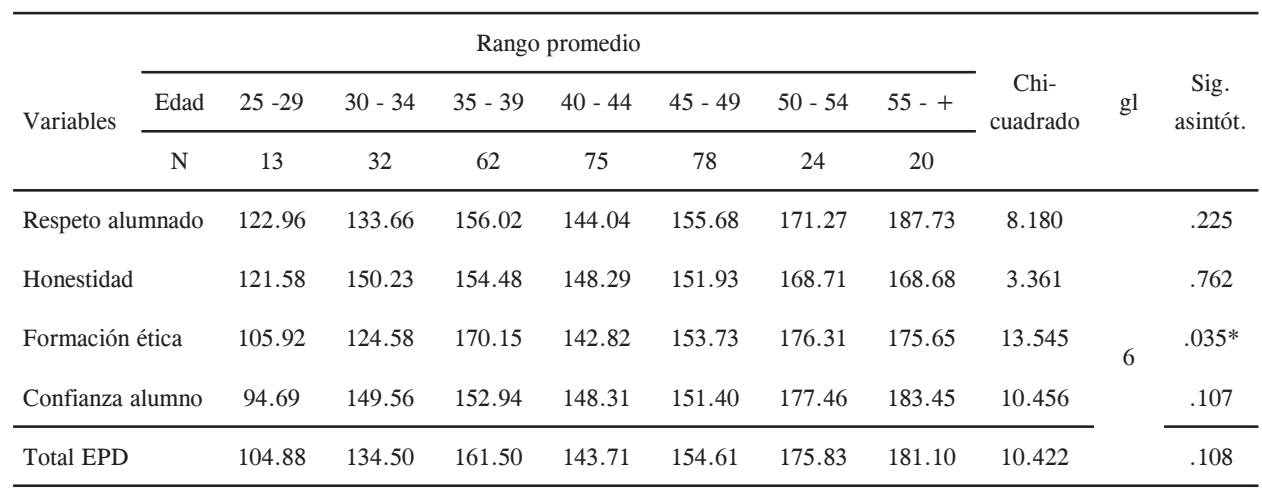

$* \mathrm{p}<.05 * * \mathrm{p}<.01 * * * \mathrm{p}<.001$

$\mathrm{n}=304$

El análisis de varianza por Rangos de Kruskall-Wallis de la Ética Profesional por Rango de Edad indica que existen diferencias estadísticas significativas solo en el caso de Formación 
ética (Chi-Cuadrado $=13.545 \mathrm{gl}=6 \mathrm{p}<.05)$ notándose que los docentes que están en los rangos de edad de 50 - 54 (Rango Promedio $=176.31$ ) y de 55 a más (Rango Promedio $=175.65)$ superan a los de $25-29($ Rango Promedio $=105.92)$, a los de 30 - 34 (Rango Promedio = 124.58), a los de 40 - 44 (Rango Promedio $=142.82$ ), a los de $45-49($ Rango Promedio $=153.73)$ y a los de $35-39($ Rango Promedio $=170.15)$.

TABLA 7. Análisis de Varianza por Rangos de Kruskal - Wallis del Desempeño Laboral Docente por Rango de Edad

\begin{tabular}{|c|c|c|c|c|c|c|c|c|c|c|c|}
\hline \multirow{3}{*}{ Variables } & \multicolumn{8}{|c|}{ Rango promedio } & \multirow{3}{*}{$\begin{array}{c}\text { Chi- } \\
\text { cuadrado }\end{array}$} & \multirow{3}{*}{$\mathrm{gl}$} & \multirow{3}{*}{$\begin{array}{c}\text { Sig. } \\
\text { asintót. }\end{array}$} \\
\hline & Edad & $25-29$ & $30-34$ & $35-39$ & $40-44$ & $45-49$ & $50-54$ & $55-+$ & & & \\
\hline & $\mathrm{N}$ & 13 & 32 & 62 & 75 & 78 & 24 & 20 & & & \\
\hline \multicolumn{2}{|l|}{ Saber conocer } & 133.92 & 138.31 & 155.06 & 153.97 & 150.90 & 169.54 & 159.60 & 2.568 & \multirow{6}{*}{6} & .861 \\
\hline \multicolumn{2}{|l|}{ Saber hacer } & 153.62 & 148.64 & 148.36 & 157.89 & 152.38 & 145.85 & 158.98 & .734 & & .994 \\
\hline \multicolumn{2}{|l|}{ Saber convivir } & 170.00 & 148.44 & 147.13 & 142.66 & 157.06 & 178.48 & 152.20 & 4.092 & & .664 \\
\hline Saber ser & & 144.12 & 144.56 & 140.36 & 153.85 & 150.99 & 190.60 & 163.38 & 6.460 & & .374 \\
\hline \multicolumn{2}{|l|}{ Saber emprender } & 147.50 & 134.75 & 148.82 & 155.43 & 152.56 & 177.42 & 154.43 & 3.496 & & .744 \\
\hline \multicolumn{2}{|c|}{ Total desempeño laboral } & 151.19 & 141.16 & 146.40 & 153.65 & 152.53 & 174.67 & 159.38 & 2.497 & & .869 \\
\hline
\end{tabular}

$* \mathrm{p}<.05 * * \mathrm{p}<.01 * * * \mathrm{p}<.001$

$\mathrm{n}=304$

El análisis de varianza por Rangos de Kruskall-Wallis del Desempeño Laboral Docente por Rango de Edad indica que no existen diferencias estadísticas significativas en caso alguno, por lo que podemos afirmar que sin importar la edad que tengan, los docentes opinan de la misma manera respecto de su Calidad Docente.

TABLA 8. Análisis descriptivo de la Ética Profesional

\begin{tabular}{ccc}
\hline Niveles & Casos & Porcentaje \\
\hline Alto & 99 & 32.6 \\
Medio & 99 & 32.6 \\
Bajo & 106 & 34.9 \\
\hline Total & 304 & 100.0 \\
\hline
\end{tabular}

$$
\mathrm{n}=304
$$

Los resultados observados en la Tabla 8 nos muestran que el $34.9 \%$ de los sujetos de la muestra presentan un bajo nivel en su ética profesional, mientras que el $32.6 \%$ está en un nivel medio y el $32.6 \%$ está en un nivel alto. 
Estos resultados son preocupantes puesto que solo el $32.6 \%$ de los docentes se declaran como personas con una buena ética profesional, mientras que el resto lo pone en duda o simplemente admite que no tiene la ética profesional que requiere la función que cumple en la sociedad.

TABLA 9. Análisis del Desempeño Laboral Docente

\begin{tabular}{ccc}
\hline Niveles & Casos & Porcentaje \\
\hline Alto & 92 & 30.3 \\
Medio & 111 & 36.5 \\
Bajo & 101 & 33.2 \\
\hline Total & 304 & 100.0 \\
\hline
\end{tabular}

$\mathrm{n}=304$

Los resultados observados en la Tabla 9 nos muestran que el $33.2 \%$ de los sujetos de la muestra presentan un bajo nivel de desempeño laboral docente, mientras que el $36.5 \%$ está en un nivel medio y el $30.3 \%$ está en un nivel alto. Estos resultados son igualmente preocupantes en la medida que los docentes asumen que no están lo suficientemente calificados para el ejercicio de su actividad profesional, esto se desprende del resultado medio y bajo que se observa en los resultados. Es necesario tomar medidas que puedan revertir esta situación y acercarnos a la ansiada calidad educativa que todas las instituciones dedicadas a la educación, deben tener.

\section{DISCUSIÓN}

De acuerdo a los resultados del análisis psicométrico de la prueba de ética profesional docente, todos los ítems forman parte de dicho inventario, y corresponden a cada una de las cuatro subescalas asignadas en el inventario. El instrumento es confiable en la medida que sus escalas obtuvieron coeficientes Alfa de Cronbach con valores que oscilan entre 0.91 y 0.94 .

En cuanto a la validez, el resultado del Análisis Factorial Exploratorio indica que la prueba está conformada por un solo factor que en general explica el $95.25 \%$ de la varianza total. Por otra parte, observamos que la medida de adecuación del muestreo de Kaiser-MeyerOlkin alcanza un valor de 0.88 que puede considerarse como adecuado, mientras que el test de esfericidad de Bartlett presenta un valor que es significativo. Estos hallazgos permiten concluir que la prueba de Ética profesional docente presenta validez de constructo.

En cuanto a la escala de Desempeño laboral docente, según los resultados del análisis de ítems, los 50 reactivos deben permanecer conformando el Cuestionario en las escalas asignadas por el autor de la versión original. Asimismo, los coeficientes alfa de Cronbach alcanzados en las cinco escalas oscilan entre 0.84 y 0.91 , lo cual indica que la escala es confiable. 
Los resultados del Análisis Factorial Exploratorio de la prueba de desempeño laboral docente indica que está conformada por un solo factor, que explica el $92.00 \%$ de la varianza total. Por otra parte, tenemos que la medida de adecuación del muestreo de Kaiser-MeyerOlkin alcanza un valor de 0.90 que puede considerarse como adecuado, mientras que el test de esfericidad de Bartlett presenta un valor que es significativo. Estos hallazgos permiten concluir que la prueba de desempeño laboral docente presenta validez de constructo.

En lo que se refiere a los resultados de la investigación alcanzados, estos nos indican la existencia de correlaciones significativas entre las variables en estudio, lo cual es un indicador de que ambas variables son realmente importantes en el funcionamiento de una institución educativa, lo cual confirmaría las formulaciones teóricas desarrolladas en el marco teórico, las mismas que afirman la importancia de ligar permanentemente estos dos aspectos en toda institución que pretende alcanzar altos niveles de calidad.

Por otra parte y a partir de estos resultados, debe quedar claro que la formación y capacitación de los docentes es una necesidad esencial, dado que el ejercicio de su función se basa en la preparación ética y técnica académica de quien lo ejerce. Se debe considerar que la ética es el ideal de la conducta humana, orientando sobre lo que es bueno y correcto y se consolida cuando se internalizan las normas sin que exista presión exógena para su cumplimiento. La ética de un profesional, y particularmente del docente, se gesta desde la formación del mismo, por ello el docente debe actuar en esta etapa, y para realizar esta labor tiene que conocer de ética y cómo debe ser su comportamiento como docente.

Desde este punto de vista González (1997), afirma que "la ética de un profesional no se adquiere en la práctica de la profesión, sino que se gesta desde la formación profesional", y es aquí donde los docentes debemos actuar, particularmente quienes tienen a su cargo la formación de los futuros docentes. Si acaso logramos darles una sólida formación ética es seguro que estaremos frente a docentes que por lo menos se esforzarán por desarrollar un trabajo de calidad.

Por otra parte, se hace necesario precisar, a partir de los resultados logrados, que trabajar con calidad y para la calidad es una preocupación que nos caracteriza actualmente, es una aspiración, una meta y tal vez una necesidad; sin embargo este deseo involucra muchos aspectos, pero tal parece que sólo fueran aspectos materiales los que se necesitan para hacer bien las cosas, en educación sobre todo, creemos que calidad es hacer las cosas de manera que sean funcionales, eficientes y eficaces pero se ha dejado de lado un punto importante; los aspectos de la formación humana, aquellos que "no se ven" porque cada persona los lleva consigo desde que inicia su proceso de formación profesional.

Es cierto, por ello, que una institución eficaz es determinante en la obtención de mejores resultados en lo académico y en lo administrativo, lo cual quiere decir que debe haber un buen nivel de organización para permitir un mejor desempeño y satisfacción en el trabajo y brindar el mejor producto o servicio a nuestros alumnos. 


\section{CONCLUSIONES}

1. Los análisis estadísticos realizados, revelan que las pruebas utilizadas en la presente investigación, Ética profesional y Desempeño Laboral Docente, presentan validez y confiabilidad.

2. Los análisis estadísticos realizados demuestran la existencia de relaciones significativas y positivas entre ética profesional y Desempeño Laboral de los docentes de las Instituciones Educativas de un distrito del cono norte de Lima.

3. El análisis de la diferencias entre los docentes varones y mujeres respecto de la ética profesional docente, indica que no existen diferencias estadísticas significativas en caso alguno.

4. El análisis de la diferencias entre los docentes varones y mujeres respecto del desempeño laboral, indica que no existen diferencias estadísticas significativas en caso alguno.

5. El análisis de Kruskal Wallis de la Ética Profesional Docente por Edad indica que existen diferencias estadísticas significativas solo en el caso de Formación ética notándose que los docentes que están en los rangos de edad de 50 - 54 y de 55 a más superan a los de 25 - 29, a los de 30 - 34, a los de 40 - 44, a los de 45 - 49 y a los de 35 - 39.

6. El análisis de Kruskal Wallis del Desempeño Laboral Docente por Edad indica que no existen diferencias estadísticas significativas en ningún caso, por lo que podemos afirmar que sin importar la edad que tengan, los docentes opinan de la misma manera respecto de su Desempeño Laboral Docente.

7. Los resultados descriptivos indican que solo el $32.6 \%$ de los docentes se declaran como personas con una adecuada ética profesional, mientras que el resto lo pone en duda o simplemente admite que su ética profesional está por debajo de lo esperado.

8. Los resultados descriptivos indican que solo un tercio de los docentes asumen que están lo suficientemente calificados para el ejercicio de su actividad profesional.

\section{REFERENCIAS BIBLIOGRÁFICAS}

1. Álvarez, M. (2001). Diseño y evaluación de programas de educación emocional. Barcelona: CISSPRAXIS Educación.

2. Alvarado, O. (1998) Administración de la Educación: Enfoque Gerencial. Lima: Universidad de Lima.

3. Altarejos, F. y otros (1998). Ética Docente. Elementos para una deontología Profesional. Barcelona: Editorial Ariel, S.A.

4. Aranguren, J. L. (1997). Ética. Madrid: Editorial Biblioteca Nueva.

5. Aristóteles (1999) La ética de Aristóteles. Traducción de Pedro Simón. Alicante: Biblioteca Virtual Miguel de Cervantes. 
6. Alcántara, J. (1993). Manual de Ética Profesional. Santiago de los Caballeros: Editorial Brens.

7. Bonete, E. (1998). La política desde la Ética. Historia de un dilema. Barcelona: Editorial Proyecto A. Ediciones.

8. Cortina, A. (1994). La Ética de las Profesiones. Pamplona: Editorial Verbo Divino.

9. Cortina, A. (1996). El quehacer ético. Editorial Santillana.

10. Cortina, A. Conill, J. (2000). 10 Palabras Clave en Ética de las Profesiones. Navarra: Editorial Verbo Divino, 13-28.

11. Cobo, J. M. (2001) Ética profesional en ciencias humanas y sociales. Madrid: Huerga Fierro Editores.

12. Delors, J. (1996). La educación encierra un tesoro. Madrid: Santillana, Ediciones UNESCO.

13. Deming, E. (1989). Calidad, productividad y competitividad: la salida de la crisis. Madrid: Ediciones Díaz de Santos.

14. Drucker, P. F. (1997). La organización del futuro. Bilbao: Deusto.

15. Escámez, J. y Gil, R. (2001). La educación en la responsabilidad. Madrid: Ediciones Paidós Ibérica S.A.

16. Fernández, J. y Hortal, A. (1994). Ética de las Profesiones. Madrid: Publicaciones de la Universidad Pontificia Comillas.

17. Filmus, D. (1995). Los condicionantes de la calidad educativa. Buenos Aires: Novedades Educativas.

18. Gimeno Sacristán, J. (1992). Comprender y transformar la enseñanza. Madrid: Morata.

19. González, J. (2000). El poder de Eros. Fundamentos y valores de ética y bioética. México, D.F.: UNAM.

20. Hernández, Fernández y Baptista (2006). Metodología de la Investigación. México: Ed. McGraw Hill.

21. Hirsch, A. y López, R. (2003). Ética profesional e identidad institucional. México: Universidad Autónoma de Sinaloa, 27-42.

22. Hortal, A. (2002) Ética General de las Profesiones. Bilbao: Desclée De Brouwer, S.A.

23. Isikawa, K. (2008). Introducción al control de calidad. Madrid: Ediciones Díaz de Santos.

24. Juran J.M, Cryna F.M, Binghan, R.S. (1999). Manual de Control de Calidad. Ed. Reverté.

25. Kerlinger, F (1998). Investigación del comportamiento. México: McGraw Hill.

26. Lafourcade, P. (1988). La evaluación como aporte a la calidad de la educación. Buenos Aires: Ed. Kapeluz

27. Luque, A., et al. (2000). Educar la tolerancia. Sevilla: Ed. Díada.

28. Mateo, J. et al. (1996). Evaluación del Profesorado de Secundaria. Barcelona: Cedecs. 
29. Manes, J.M. (2005). Modelo Integral de Calidad en Educación, MICE. Ponencia del Presidente del Foro Interamericano de Administración de la Educación (FIAE) en el "Foro Internacional de Modelos de Calidad y su aplicación en preescolar, básica y media", organizado por CONACED, Bogotá.

30. Martínez, M.J. (1999). Gestión de la calidad en la empresa. En: Álvarez, J.L. Lo que se aprende en los mejores MBA. Barcelona: Ediciones Gestión 2000, p. 573-616.

31. Mintzberg, H. (1991). La estructuración de las organizaciones. Barcelona: Ariel Economía.

32. Marchesi, A., Martín, E. (1998). Calidad de la enseñanza en tiempos de cambio. Madrid: Alianza Editorial.

33. Sánchez, H y Reyes, C. (2002). Metodología y Diseños en la Investigación Científica. Lima: URP. Editorial Universitaria.

34. Toulouse, G. (2003) Mirada sobre la ética de las ciencias. Madrid, Ediciones del Laberinto.

35. Trías, E. (2000). Ética y Condición Humana. Barcelona: Editorial Península.

36. Valdés, H. (2000) Evaluación del Desempeño Docente. Ponencia presentada al Encuentro Iberoamericano sobre Evaluación del Desempeño Docente. México

37. Yegres, A. (1999). Ética y formación docente. Venezuela: Editorial Gerardo Toro. 